P-224 OCCUPATIONAL RADIATION EXPOSURE AND INFLAMMATORY BIOMARKERS RELATED TO CIRCULATORY DISEASES AMONG MALE INTERVENTIONAL MEDICAL WORKERS

${ }^{1}$ Ye Jin Bang, Seulki Ko, Kyoung-Mu Lee, Seung-Ah Choe, Byung-Chul Chun, Won Jin Lee. ${ }^{1}$ Korea University, Republic of Korea

10.1136/OEM-2021-EPI.239

Introduction A few epidemiologic studies have reported increased risk of circulatory disease among population exposed to ionizing radiation although the mechanism has not been established.

Objectives We investigated the association between occupational radiation exposure and inflammatory biomarkers in medical workers at interventional radiology departments.

Methods We conducted a field survey for interventional medical workers in 2017. Individual radiation dose was linked to the National Dose Registry in 1996-2017. Twenty-three inflammatory biomarkers related to circulatory diseases were measured using by multiplex immunoassays in blood plasma. Multiple linear regression analysis was used to analyze the associations between radiation exposure and inflammatory biomarkers adjusted for potential confounders.

Results A total of 73 male medical workers (52 radiologists, 21 radiologic technologists) were included in this study and they have been worked for a mean of 11.1 years at the department of interventional radiology. The mean cumulative badge dose was $22.5 \mathrm{mSv}$, which ranged from the 0.01 to $109.3 \mathrm{mSv}$. The pro-inflammatory cytokines IFN- $\beta$, IFN\&gamma; and anti-inflammatory cytokines IL-1ra, IL-4 were significantly decreased with cumulative occupational radiation dose (coefficient $=-0.388$ per $10 \mathrm{mSv},-0.080$ per $10 \mathrm{mSv},-0.564$ per $10 \mathrm{mSv},-0.068$ per $10 \mathrm{mSv}$, respectively), whereas endothelial cell adhesion molecules E-selectin and pro-inflammatory cytokines MIF showed significantly increased association (coefficient $=0.058$ per $10 \mathrm{mSv}, 0.189$ per $10 \mathrm{mSv}$, respectively). The association between radiation dose and the level of biomarkers were similar by job titles.

Conclusion This study suggests that low-dose occupational radiation exposure was associated with a few inflammatory biomarkers related with circulatory diseases among interventional medical workers. However, given some limitations, further investigation with larger population are warranted.

\section{P-227 CANCER OUTCOMES OF EPIDEMIOLOGICAL STUDIES AMONG PETROLEUM WORKERS AND RESIDENTS LIVING IN OIL PRODUCING COMMUNITIES: A SYSTEMATIC REVIEW AND META-ANALYSIS}

${ }^{1}$ Felix Onyije, Bayan Hosseini, Kayo Togawa, Joachim Schuz, Ann Olsson. 'International Agency for Research on Cancer, France

\subsection{6/OEM-2021-EPI.240}

Introduction Petroleum extraction and refining are major sources of various occupational carcinogens and of air pollution and may therefore contribute to the global cancer burden.

Objective The objective of this systematic review was to evaluate the cancer risk in workers employed in petroleum industries and residents living in oil producing communities.

Methods Relevant studies were identified and retrieved through PubMed and Web of Science databases. Summary effect size (ES) and 95\% confidence intervals (CI) were analysed using random effect models, and heterogeneity across studies was assessed (I2).

Results Overall, petroleum industry work was associated with an increased risk of mesothelioma (ES $=2.09$, CI: 1.58 2.76), skin melanoma ( $\mathrm{ES}=1.34$, CI: 1.06-1.70 multiple myeloma ( $\mathrm{ES}=1.81, \mathrm{CI}: 1.28-2.55)$, and cancers of the prostate $(\mathrm{ES}=1.13, \mathrm{Cl}: 1.05-1.22)$ and urinary bladder $(\mathrm{ES}=$ 1.25, CI: 1.09-1.43) and a decreased risk of cancers of the esophagus, stomach, colon, rectum, and pancreas. Offshore petroleum work was associated with an increased risk of lung cancer $(\mathrm{ES}=1.20 ; 95 \% \mathrm{CI}: 1.03-1.39)$ and leukemia $(\mathrm{ES}=$ 1.47; 95\% CI: 1.12-1.92) in stratified analysis. Residential proximity to petroleum facilities was associated with childhood leukemia ( $\mathrm{ES}=1.90, \mathrm{CI}:$ 1.34-2.70).

Conclusion Many of the associations however appear to be due to factors other than those directly emerging from the petroleum production, including the inverse associations. Improved exposure assessment is needed in further studies to identify the drivers of the observed cancer risks. In particular, there is a need for targeted studies in under-researched areas of high petroleum production with presumably higher exposures. An international consortium guiding new generation studies in Africa, the Middle East and Asia, to harmonize study protocols and exposure assessments, may be the most promising way forward.

\section{P-228 OCCUPATIONAL EXPOSURE TO RADIOFREQUENCY ELECTROMAGNETIC FIELDS AND RISK OF CANCER: PRELIMINARY DATA FROM THE ITALIAN RESEARCH PROJECT BRIC 2018 - ID 06}

${ }^{1}$ Alberto Modenese, Giulia Bravo, Carlo Grandi, Mauro Biffoni, Fabriziomaria Gobba. ${ }^{1}$ University of Modena \& Reggio Emilia, Italy

\subsection{6/OEM-2021-EPI.241}

Introduction The possible role of Radiofrequency electromagnetic fields (RF-EMF) exposure as potential human carcinogen has been extensively studied considering mobile phones use, while until now less effort was devoted to an evaluation of the risk related to other occupational exposures.

Objectives The aim of the research is to evaluate the evidence on the possible association between RF-EMF exposure in various occupational activities and carcinogenic effects in published epidemiologic studies.

Methods A systematic review of human epidemiologic studies investigating carcinogenic effects in workers occupationally exposed to RF-EMF is ongoing within the Italian research project BRIC 2018 - ID 06, supported by the Italian Workers' Compensation Authority (INAIL). The protocol of the study has been registered in the PROSPERO database (CRD42020200202).

Results The preliminary results of the review indicate that, even if various types of cancers as blood and lymphatic neoplasms, uveal melanoma, testicular, breast, lung and skin cancers were also evaluated, the majority of the studies focus on the possible association between occupational RF-EMF exposure and brain tumors. One of the major problems of research, observed in most of the studies, is the evaluation of exposure: the methods applyed for RF-EMF exposure classification and assessment have several limits, possibly jeopardizing aggregate quantitative analyses (as, e.g., pooling Odds Ratios 